\title{
ON FINITE FUNCTIONS WITH NON-TRIVIAL ARITY GAP
}

\author{
SLAVCHO SHTRAKOV AND JÖRG KOPPITZ
}

\begin{abstract}
Given an $n$-ary $k$-valued function $f, g a p(f)$ denotes the minimal number of essential variables in $f$ which become fictive when identifying any two distinct essential variables in $f$.

We particularly solve a problem concerning the explicit determination of $n$-ary $k$-valued functions $f$ with $2 \leq g a p(f) \leq n \leq k$. Our methods yield new combinatorial results about the number of such functions.
\end{abstract}

\section{INTRODUCTION}

Given a function $f$, the essential variables in $f$ are defined as variables which occur in $f$ and weigh with the values of that function. The number of essential variables is an important measure of complexity for discrete functions. Some deep results characterizing such variables and sets of essential variables are known (2, 3, 8, 9, 10]). Similar problems about terms in universal algebra are discussed in [7, 11, 12] and about tree automata in [13. So, any opportunity to reduce the number of essential variables in discrete functions is an important procedure in theoretical and applied computer science and modeling. There are two ways to decrease this number - by replacing some variables in function with constants or with other variables (i.e. with identification of variables).

M. Couceiro and E. Lehtonen classify finite valued functions on a finite set $A$ in terms of their arity gap $(\underline{6})$. The aim of the present paper is the representation and description of such functions. We shall use the well known fact that each $n$-ary function $f: A^{n} \rightarrow A$ can be represented as sums of conjunctions.

In Section 2 we introduce the basic definitions and some preliminary results concerning $k$-valued functions.

In Section 3 we study the essential arity gap of functions. Here a complete description of the functions in $k$-valued logic depending essentially on all of its $n$ variables whose essential arity gap is equal to $p$ with $2<p \leq n \leq k$ is obtained.

In Section 4 we consider the class $G_{2, k}^{n}$ of $n$-ary $k$-valued functions which have essential arity gap equal to 2 when $k>2$. This class is presented as union of two subclasses which are investigated.

In Section 5 we discuss a special class of the ternary $k$-valued functions which have essential arity gap equal to 2 . The number of functions in this class is found.

2000 Mathematics Subject Classification. Primary: 03G25; Secondary: 05E05

ACM-Computing Classification System (1998) : G.2.0.

Key words and phrases. essential variable, identification minor, essential arity gap.

The research was supported by the DFG project KO 1446/3-1. 


\section{Preliminaries}

Let $k>2$ be a natural number. Denote by $K=\{0,1, \ldots, k-1\}$ the set (ring) of remainders modulo $k$. A function (operation) on $K$ is a mapping $f: K^{n} \rightarrow K$ where $n$ is a natural number, called the arity of $f$. The set of the all such functions is denoted by $P_{k}^{n}$. Operations from $P_{2}^{n}$ are called Boolean functions.

Let $X_{n}=\left\{x_{1}, \ldots, x_{n}\right\}$ be the set of $n$ variables and $f \in P_{k}^{n}$ be a $k$-valued function.

Definition 2.1. A variable $x_{i}$ is called essential in $f$, or $f$ essentially depends on $x_{i}$, if there exist values $a_{1}, \ldots, a_{n}, b \in K$, such that

$$
f\left(a_{1}, \ldots, a_{i-1}, a_{i}, a_{i+1}, \ldots, a_{n}\right) \neq f\left(a_{1}, \ldots, a_{i-1}, b, a_{i+1}, \ldots, a_{n}\right) .
$$

The set of all essential variables in a function $f$ is denoted by $E s s(f)$ and the number of its essential variables is denoted by ess $(f)=|\operatorname{Ess}(f)|$. The variables from $X_{n}$ which are not essential in $f \in P_{k}^{n}$ are called fictive and the set of fictive variables in $f$ is denoted by $F i c(f)$.

Let $x_{i}$ and $x_{j}$ be two distinct essential variables in $f$. We say that the function $g$ is obtained from $f \in P_{k}^{n}$ by the identification of the variable $x_{i}$ with $x_{j}$, if

$$
g\left(a_{1}, \ldots, a_{i-1}, a_{i}, a_{i+1}, \ldots, a_{n}\right)=f\left(a_{1}, \ldots, a_{i-1}, a_{j}, a_{i+1}, \ldots, a_{n}\right),
$$

for all $\left(a_{1}, \ldots, a_{n}\right) \in K^{n}$.

Briefly, when $g$ is obtained from $f$, by identification of the variable $x_{i}$ with $x_{j}$, we will write $g=f_{i \leftarrow j}$ and $g$ is called the identification minor of $f$ and $\operatorname{Min}(f)$ denotes the set of all identification minors of $f$.

We shall allow formation of identification minors when $x_{i}$ or $x_{j}$ are not essential in $f$, also. Such minors of $f$ are called trivial and they do not belong to $\operatorname{Min}(f)$. For instance, if $x_{i}$ does not occur in $f$, then $f_{i \leftarrow j}:=f$.

Remark 2.1. Let $i$ and $j$ be two natural numbers with $1 \leq j, i \leq n, i \neq j$. Then we have:

(i) ess $\left(f_{i \leftarrow j}\right) \leq e s s(f)$, because $x_{i} \notin E s s\left(f_{i \leftarrow j}\right)$, even though it might be essential in $f$.

(ii) If $x_{j}$ is an essential variable in $f$ then $\operatorname{Ess}\left(f_{i \leftarrow j}\right) \subseteq E s s(f)$.

Definition 2.2. Let $f \in P_{k}^{n}$ be an $n$-ary $k$-valued function. Then the essential arity gap (briefly arity gap or gap) of $f$ is defined as follows

$$
g a p(f):=e s s(f)-\max _{g \in \operatorname{Min}(f)} \operatorname{ess}(g) .
$$

We say that the function $f$ has non-trivial arity gap if $\operatorname{gap}(f) \geq 2$.

We let $G_{p, k}^{m}$ denote the set of all functions in $P_{k}^{n}$ which essentially depend on $m$ variables whose arity gap is equal to $p$ i.e. $G_{p, k}^{m}=\left\{f \in P_{k}^{n} \mid \operatorname{ess}(f)=m \& g a p(f)=\right.$ $p\}$, with $m \leq n$.

The set of all $n$-ary $k$-valued functions which essentially depend on $m$ variables is denoted by $P_{m, k}^{n}$, i.e. $P_{m, k}^{n}=\left\{f \in P_{k}^{n} \mid \operatorname{ess}(f)=m\right\}, m \leq n$.

An upper bound of $g a p(f)$ for Boolean functions is found by K.Chimev, A. Salomaa and O. Lupanov [4, 10, 9]. It is proved that $\operatorname{gap}(f) \leq 2$, when $f \in$ $P_{2}^{n}, n \geq 2$. 
This result is generalized for arbitrary finite valued functions in [5]. It is shown that $g a p(f) \leq k$ for all $f \in P_{k}^{n}$.

In [5] the Boolean functions whose arity gap is equal to 2 are described. In [14] the class $G_{2,2}^{n}$ is investigated, also. Several combinatorial results concerning the number of the functions in this class are obtained.

The case $2 \leq p \leq k<n$ is fully described in 15 where it is proved that $\operatorname{gap}(f) \leq 2$ and if $f \in G_{2, k}^{n}$ then $f$ is a totally symmetric function.

So, in the present paper we shall pay attention to the case $2<k$ and $n \leq k$.

We show that if $f \in G_{p, k}^{n}, 2<p \leq n \leq k$ then $f=h \oplus g$ where $\operatorname{ess}(h)=n-p$ and $g \in G_{n, k}^{n}$ which arises in this way, solving the following problem:

For each $1 \leq p \leq k$, determine explicitly the functions $f \in P_{k}^{n}$ whose arity gap is

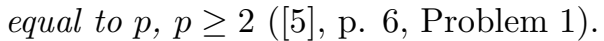

Let $m \in N, 0 \leq m \leq k^{n}-1$ be an integer. It is well known that for every $k, n \in N, k \geq 2$ there is an unique finite sequence $\left(\alpha_{1}, \ldots, \alpha_{n}\right) \in K^{n}$ such that

$$
m=\alpha_{1} k^{n-1}+\alpha_{2} k^{n-2}+\ldots+\alpha_{n} .
$$

The equation (11) is known as the representation of $m$ in $k$-ary positional numerical system. One briefly writes $m=\overline{\alpha_{1} \alpha_{2} \ldots \alpha_{n}}$ instead (1).

Given a variable $x$ and $\alpha \in K, x^{\alpha}$ is an important function defined by:

$$
x^{\alpha}=\left\{\begin{array}{lll}
1 & \text { if } & x=\alpha \\
0 & \text { if } & x \neq \alpha .
\end{array}\right.
$$

In this paper we shall use sums of conjunctions (SC) for representation of functions in $P_{k}^{n}$. This is the most natural representation of the functions in finite algebras. It is based on so called operation tables of the functions.

Theorem 2.1. Each function $f \in P_{k}^{n}$ can be uniquely represented in $S C$-form as follows

$$
f=a_{0} \cdot x_{1}^{0} \ldots x_{n}^{0} \oplus \ldots \oplus a_{m} \cdot x_{1}^{\alpha_{1}} \ldots x_{n}^{\alpha_{n}} \oplus \ldots \oplus a_{k^{n}-1} \cdot x_{1}^{k-1} \ldots x_{n}^{k-1}
$$

with $m=\overline{\alpha_{1} \ldots \alpha_{n}}, a_{m} \in K$, where $\oplus "$ and "." are the operations addition and multiplication modulo $k$ in the ring $K$.

A fact we shall use repeatedly is this: A variable $x_{i}$ is fictive (inessential) in the function $f \in P_{k}^{n}$, if and only if

$$
f\left(x_{1}, \ldots, x_{n}\right)=x_{i}^{0} \cdot f_{1} \oplus x_{i}^{1} \cdot f_{2} \ldots \oplus x_{i}^{k-1} \cdot f_{k},
$$

with $f_{1}=f_{2}=\ldots=f_{k}$ and $x_{i} \notin E s s\left(f_{j}\right)$, where $f_{j}$ are $n$-1-ary $k$-valued functions with set of variables $\left\{x_{1}, \ldots, x_{i-1}, x_{i+1}, \ldots, x_{n}\right\}$ for $j=1,2, \ldots, k$.

Consequently, if $f, g \in P_{k}^{n}, x_{i} \in F i c(f)$ and $x_{i} \in F i c(g)$, then $x_{i} \in F i c(f \oplus g)$.

Let us note that if $f_{i \leftarrow j}=g_{i \leftarrow j}$ and $\alpha_{i}=\alpha_{j}$ then $f\left(\alpha_{1}, \ldots, \alpha_{j}, \ldots, \alpha_{i}, \ldots, \alpha_{n}\right)=$ $g\left(\alpha_{1}, \ldots, \alpha_{j}, \ldots, \alpha_{i}, \ldots, \alpha_{n}\right)$.

Lemma 2.1. Let $f$ and $g$ be two $k$-valued functions, depending essentially on $n, n \geq k+1$ variables. If $f_{i \leftarrow j}=g_{i \leftarrow j}$ for all $i, j$ with $1 \leq j, i \leq n$ and $i \neq j$, then $f=g$.

Proof. Let $\left(\alpha_{1}, \alpha_{2}, \ldots, \alpha_{n}\right) \in K^{n}$ be an arbitrary $n$-tuple of integers from $K$. Since $n \geq k+1$ it follows that there exist two natural numbers $i, j$ with $1 \leq j<i \leq n$ and $\alpha_{i}=\alpha_{j}$. Then $f_{i \leftarrow j}=g_{i \leftarrow j}$ implies

$$
f\left(\alpha_{1}, \ldots, \alpha_{j}, \ldots, \alpha_{i}, \ldots, \alpha_{n}\right)=g\left(\alpha_{1}, \ldots, \alpha_{j}, \ldots, \alpha_{i}, \ldots, \alpha_{n}\right) .
$$


Consequently $f=g$.

Example 2.1. Let us consider the functions $f=x_{1}^{0} x_{2}^{0} x_{3}^{0} \oplus x_{1}^{1} x_{2}^{0} x_{3}^{2}$ and $g=x_{1}^{0} x_{2}^{0} x_{3}^{0} \oplus$ $x_{1}^{0} x_{2}^{1} x_{3}^{2}$ from $P_{3}^{3}$. It is not too hard to show that $f_{i \leftarrow j}=g_{i \leftarrow j}=x_{j}^{0} x_{m}^{0}$, where $m \in\{1,2,3\} \backslash\{i, j\}$ for all $i, j$ with $1 \leq j, i \leq 3$ and $i \neq j$. On the other hand we have $f \neq g$. This example shows that $n \geq k+1$ is an essential condition in Lemma 2.1.

Lemma 2.2. If $x_{i} \notin E s s(f)$ and $f \in P_{k}^{n}$ then $f=f_{i \leftarrow j}$, for all $j \in\{1 \ldots n\}$, $i \neq j$.

Proof. Without loss of generality assume that $j=1$ and $i=2$. Then we have

$$
f\left(\alpha_{1}, \alpha_{2}, \alpha_{3}, \ldots, \alpha_{n}\right)=f\left(\alpha_{1}, \beta_{2}, \alpha_{3}, \ldots, \alpha_{n}\right),
$$

for all $\alpha_{1}, \alpha_{2}, \alpha_{3}, \ldots, \alpha_{n}, \beta_{2} \in K$. Let $\gamma_{1}, \gamma_{2}, \gamma_{3}, \ldots, \gamma_{n} \in K$ be arbitrary $n$ integers from $K$. Then we have

$$
f\left(\gamma_{1}, \gamma_{1}, \gamma_{3}, \ldots, \gamma_{n}\right)=f\left(\gamma_{1}, \gamma_{2}, \gamma_{3}, \ldots, \gamma_{n}\right) .
$$

Consequently $f=f_{2 \leftarrow 1}$.

Lemma 2.2 implies that if $x_{i} \notin E s s(f)$ then $\operatorname{Ess}(f)=\operatorname{Ess}\left(f_{i \leftarrow j}\right)$, for all $j \in$ $\{1 \ldots n\}$ with $i \neq j$.

\section{EsSEnTIAL ARITY GAP OF $k$-VALUED FUNCTIONS}

We are going to study the $n$-ary $k$-valued functions whose arity gap is equal to $n$. The set of all strings over $K$ with length $m, m \geq 1$ will be denoted by $K^{m}$.

Given two natural numbers $k, n \geq 2, E q_{k}^{n}$ denotes the set of all strings over $K=\{0,1, \ldots, k-1\}$ with length $n$ which have at least two equal letters i.e.

$$
E q_{k}^{n}:=\left\{\alpha_{1} \ldots \alpha_{n} \in K^{n} \mid \alpha_{i}=\alpha_{j}, \text { for some } i, j \leq n, i \neq j\right\} .
$$

Lemma 3.1. If $f \in G_{n, k}^{n}$ and $2 \leq n \leq k$, then $f\left(\alpha_{1}, \ldots, \alpha_{n}\right)=f(0, \ldots, 0)$ for all $\alpha_{1} \ldots \alpha_{n} \in E q_{k}^{n}$.

Proof. Let $\alpha_{1} \alpha_{2} \ldots \alpha_{n}$ be an arbitrary string from $E q_{k}^{n}$. Without loss of generality let us assume that $\alpha_{1}=\alpha_{2}$. Since $f \in G_{n, k}^{n}$ it follows that the function $f_{2 \leftarrow 1}=f\left(x_{1}, x_{1}, x_{3}, \ldots, x_{n}\right)$ does not essentially depend on any of its variables $x_{1}, x_{3}, \ldots, x_{n}$. So, we have $f\left(\alpha_{1}, \alpha_{2}, \alpha_{3}, \ldots, \alpha_{n}\right)=f\left(\alpha_{1}, \alpha_{1}, \alpha_{3}, \ldots, \alpha_{n}\right)=$ $f(0, \ldots, 0)$.

Theorem 3.1. Let $f \in P_{k}^{n}$, be a function which depends essentially on all of its $n$ variables and $2 \leq n \leq k$. Then $f \in G_{n, k}^{n}$ if and only if it can be represented as follows

$$
f=\left[\bigoplus_{\beta_{1} \ldots \beta_{n} \notin E q_{k}^{n}} a_{r} \cdot x_{1}^{\beta_{1}} \ldots x_{n}^{\beta_{n}}\right] \oplus a_{0} \cdot\left[\bigoplus_{\alpha_{1} \ldots \alpha_{n} \in E q_{k}^{n}} x_{1}^{\alpha_{1}} \ldots x_{n}^{\alpha_{n}}\right],
$$

where $r=\overline{\beta_{1} \ldots \beta_{n}}$ and at least two among the coefficients

$$
\left\{a_{0}\right\} \cup\left\{a_{r} \mid r=\overline{\beta_{1} \ldots \beta_{n}}, \& \beta_{1} \ldots \beta_{n} \notin E q_{k}^{n}\right\}
$$

are distinct. 
Proof. " $\Rightarrow$ Let $f \in G_{n, k}^{n}$ be represented in its SC-form as follows

$$
f=\bigoplus_{m=0}^{k^{n-1}} a_{m} \cdot x_{1}^{\beta_{1}} \ldots x_{n}^{\beta_{n}} \quad \text { where } \quad m=\overline{\beta_{1} \ldots \beta_{n}} .
$$

By Lemma 3.1 we have $f\left(\alpha_{1}, \ldots, \alpha_{n}\right)=f(0, \ldots, 0)=a_{0}$ for $\alpha_{1} \ldots \alpha_{n} \in E q_{k}^{n}$. This shows that $f$ has to be in the form (3.1). Moreover, at least two among the coefficients $\left\{a_{0}\right\} \cup\left\{a_{r} \mid r=\overline{\beta_{1} \ldots \beta_{n}} \& \beta_{1} \ldots \beta_{n} \in E q_{k}^{n}\right\}$ are distinct.

$" \Leftarrow$ " Let $f$ be represented in the form (3). Then $f$ depends essentially on all of its variables since at least two among the coefficients $\left\{a_{0}\right\} \cup\left\{a_{r} \mid r=\right.$ $\left.\overline{\beta_{1} \ldots \beta_{n}}, \& \beta_{1} \ldots \beta_{n} \notin E q_{k}^{n}\right\}$, are distinct. We have to prove that for all $i, j$ with $1 \leq j, i \leq n$ and $i \neq j$ the functions

$$
f_{i \leftarrow j}=a_{0} . \bigoplus_{\beta_{i}=\beta_{j}} x_{1}^{\beta_{1}} \ldots x_{j}^{\beta_{j}} \ldots x_{i}^{\beta_{i}} \ldots x_{n}^{\beta_{n}}
$$

do not depend on any of their variables.

Without loss of generality let us prove this for $j=1$ and $i=2$. Then

$$
f_{2 \leftarrow 1}=a_{0} \cdot\left(x_{1}^{0} \oplus x_{1}^{1} \oplus \ldots \oplus x_{1}^{k-1}\right)\left(\bigoplus_{\beta_{3} \ldots \beta_{n} \in K^{n-2}} x_{3}^{\beta_{3}} \ldots x_{n}^{\beta_{n}}\right)=a_{0} \cdot 1 \cdot 1=a_{0} .
$$

Hence $\operatorname{ess}\left(f_{2 \leftarrow 1}\right)=0$. This completes the proof of the theorem.

Corollary 3.1. For each $k, k \geq 3$ the functions

$$
p_{k}\left(x_{1}, \ldots, x_{k}\right)=\bigoplus_{\alpha_{1} \ldots \alpha_{k} \notin E q_{k}^{k}} a_{m} \cdot x_{1}^{\alpha_{1}} \ldots x_{k}^{\alpha_{k}},
$$

with $a_{m} \in K$, have the essential arity gap $k$, excluding when all $a_{m}$ 's are equal to 0 and $p_{k}\left(x_{i}=x_{j}\right)=0$ for all $1 \leq i, j \leq n$ with $i \neq j$.

Theorem 3.2. If $2 \leq n \leq k$ then

$$
\left|G_{n, k}^{n}\right|=k\left(\left(\begin{array}{c}
k \\
n
\end{array}\right) \cdot n !+1\right)-k .
$$

Proof. The number of coefficients $a_{i}$ in (3) is equal to $\left(\begin{array}{l}k \\ n\end{array}\right) \cdot n !+1$ and they can be chosen in $k^{\left[\left(\begin{array}{l}k \\ n\end{array}\right) \cdot n !+1\right]}$ ways. There are $k$ "forbidden" cases, when $a_{0}$ and $a_{r}$ 's in (3) are the same.

Lemma 3.2. Let $f \in P_{k}^{n}$ be a k-valued function. If $x_{i} \notin E s s\left(f_{u \leftarrow v}\right)$, with $1 \leq$ $i, u, v \leq n, u \neq v$ and $i \notin\{u, v\}$ then $f_{u \leftarrow v}=\left[f_{i \leftarrow j}\right]_{u \leftarrow v}$, for all $j, j \in\{1 \ldots n\}$, $j \neq i$.

Proof. Suppose with no loss of generality that $u=2, v=1$ and $i=3$. Then $f_{2 \leftarrow 1}=f\left(x_{1}, x_{1}, x_{3}, x_{4}, \ldots, x_{n}\right)$ and from $x_{3} \notin \operatorname{Ess}\left(f_{2 \leftarrow 1}\right)$ we have

$$
f\left(x_{1}, x_{1}, \alpha, x_{4}, \ldots, x_{n}\right)=f\left(x_{1}, x_{1}, \beta, x_{4}, \ldots, x_{n}\right)
$$

for all $\alpha, \beta \in K$.

Let $j \in\{1, \ldots, n\}$ and $j \neq 3$. Then we have

$$
\left[f_{3 \leftarrow j}\right]_{2 \leftarrow 1}=f\left(x_{1}, x_{1}, x_{j}, x_{4}, \ldots, x_{n}\right)=f\left(x_{1}, x_{1}, x_{3}, x_{4}, \ldots, x_{n}\right)=f_{2 \leftarrow 1} .
$$

Lemma 3.3. Let $f \in P_{k}^{n}$ be a $k$-valued function. If $x_{v} \notin E s s\left(f_{u \leftarrow v}\right)$ for some $u, v \leq n$, then $f_{u \leftarrow v}=\left[f_{v \leftarrow j}\right]_{u \leftarrow j}=\left[f_{u \leftarrow j}\right]_{v \leftarrow j}$, for all $j, j \in\{1 \ldots n\}, j \neq u, v$. 
Proof. Without loss of generality assume that $u=2$ and $v=1$.

Then $f_{2 \leftarrow 1}=f\left(x_{1}, x_{1}, x_{3}, \ldots, x_{n}\right)$ and from $x_{1} \notin E s s\left(f_{2 \leftarrow 1}\right)$ we obtain

$$
f_{2 \leftarrow 1}=f\left(\alpha, \alpha, x_{3}, \ldots, x_{n}\right)=f\left(\beta, \beta, x_{3}, \ldots, x_{n}\right)
$$

for all $\alpha, \beta \in K$.

Let $j>2$ and by symmetry, we may assume $j=3$. Then we obtain

$$
\begin{gathered}
{\left[f_{1 \leftarrow 3}\right]_{2 \leftarrow 3}=\left[f\left(x_{3}, x_{2}, x_{3}, x_{4}, \ldots, x_{n}\right)\right]_{2 \leftarrow 3}=f\left(x_{3}, x_{3}, x_{3}, x_{4}, \ldots, x_{n}\right)=} \\
=f\left(\alpha, \alpha, x_{3}, \ldots, x_{n}\right)=f_{2 \leftarrow 1} .
\end{gathered}
$$

We are going to describe the basic properties of the functions $f$ whose arity gap is non-trivial i.e. $\operatorname{gap}(f)=p$ with $2 \leq p<n \leq k$, in the rest of the paper.

Theorem 3.3. Let $2<p<n \leq k$. Then for each $f \in G_{p, k}^{n,}$ there is a function $h \in P_{k}^{n}$ with

(i) $\operatorname{ess}(h)=n-p$;

(ii) $f_{i \leftarrow j}=h$ for all $1 \leq i, j \leq n$ with $i \neq j$ and $x_{i} \in$ Fic $(h)$.

Moreover, for all $1 \leq u, v \leq n$ with $v \neq u$ and $x_{v} \in$ Fic $\left(f_{u \leftarrow v}\right)$ holds $f_{i \leftarrow j}=f_{u \leftarrow v}$ for all $1 \leq i, j \leq n$ with $i \neq j$ and $x_{i} \in F i c\left(f_{u \leftarrow v}\right)$ as well as ess $\left(f_{u \leftarrow v}\right)=n-p$.

Proof. Let $f \in G_{p, k}^{n}$ and $1 \leq i, j \leq n$ with $i \neq j$ and $e s s\left(f_{i \leftarrow j}\right)=n-p$. Let us set $h=f_{i \leftarrow j}$.

First, we shall prove that there are $1 \leq r, s \leq n$ with $r \neq s$, ess $\left(f_{r \leftarrow s}\right)=n-p$ and $x_{s} \notin E s s\left(f_{r \rightarrow s}\right)$.

If $x_{j} \notin E s s\left(f_{i \leftarrow j}\right)$ we are done in this part of the proof.

Furthermore, let us assume that $x_{j} \in \operatorname{Ess}\left(f_{i \leftarrow j}\right)$. Since $n>p>2$, i.e. $n>3$ there are $1 \leq r, s \leq n$ with $r \neq s$ and $x_{r}, x_{s} \in$ Fic $(h) \backslash\left\{x_{i}\right\}$. We shall prove that $e s s\left(f_{r \leftarrow s}\right)=n-p$ and $x_{s} \notin E s s\left(f_{r \rightarrow s}\right)$.

By Lemma 3.2, we have

$$
f_{i \leftarrow j}=\left[f_{r \leftarrow s}\right]_{i \leftarrow j} .
$$

This gives $n-p=e s s\left(f_{i \leftarrow j}\right) \leq e s s\left(f_{r \leftarrow s}\right) \leq n-p$ since $g a p(f)=p$, i.e. ess $\left(f_{r \leftarrow s}\right)=$ $n-p$. Further, let $x_{m} \in E s s(h) \backslash\left\{x_{j}\right\}$. Assume that $x_{m} \notin E s s\left(f_{r \rightarrow s}\right)$. Then

$$
f_{r \leftarrow s}=\left[f_{r \leftarrow s}\right]_{m \leftarrow i} \text { and thus } \quad\left[f_{r \leftarrow s}\right]_{i \leftarrow j}=\left[\left[f_{r \leftarrow s}\right]_{m \leftarrow i}\right]_{i \leftarrow j} .
$$

Moreover, $h=h_{r \leftarrow s}$ since $x_{r} \notin E s s(h)$. Because of $r \neq i$, we have

$$
h_{r \leftarrow s}=\left[f_{r \leftarrow s}\right]_{i \leftarrow j} \quad \text { and thus } \quad h=\left[\left[f_{r \leftarrow s}\right]_{m \leftarrow i}\right]_{i \leftarrow j} .
$$

Since $x_{m} \neq x_{j}$ we have $x_{m} \notin E s s\left(\left[\left[f_{r \leftarrow s}\right]_{m \leftarrow i}\right]_{i \leftarrow j}\right)$, i.e. $x_{m} \notin E s s(h)$, which is a contradiction. This shows that

$$
\operatorname{Ess}(h) \backslash\left\{x_{j}\right\} \subseteq E s s\left(f_{r \leftarrow s}\right) \quad \text { and thus } \quad F i c\left(f_{r \leftarrow s}\right) \subseteq\left\{x_{j}\right\} \cup F i c(h) .
$$

Now let us prove that $x_{s} \notin E s s\left(f_{r \leftarrow s}\right)$. For suppose this were not true i.e. $x_{s} \in \operatorname{Ess}\left(f_{r \leftarrow s}\right)$. Because of $n-p=e s s\left(f_{r \leftarrow s}\right)=e s s\left(f_{i \leftarrow j}\right)$, then $x_{s} \notin F i c\left(f_{r \leftarrow s}\right)$ implies Fic $\left(f_{r \leftarrow s}\right)=\left(\left\{x_{j}\right\} \cup F i c(h)\right) \backslash\left\{x_{s}\right\}$. This provides $x_{i}, x_{j} \in F i c\left(f_{r \leftarrow s}\right)$. Then we have $f_{r \leftarrow s}=\left[f_{r \leftarrow s}\right]_{i \leftarrow j}$. Moreover, $h=h_{r \leftarrow s}$. Since $r \neq i$, we have $\left[f_{r \leftarrow s}\right]_{i \leftarrow j}=$ $h_{r \leftarrow s}$. Altogether, this provides $f_{r \leftarrow s}=h$. This shows that Fic $(h)=F i c\left(f_{r \leftarrow s}\right)$, and in particular, $x_{j} \in \operatorname{Fic}(h)$ which is a contradiction. Hence $x_{s} \notin E s s\left(f_{r \leftarrow s}\right)$.

Second, we shall prove that $h=f_{u \leftarrow v}$ for all $1 \leq u, v \leq n$ with $u \neq v$ and $x_{u} \in \operatorname{Fic}(h)$.

Let $1 \leq r, s \leq n$ with $r \neq s$ and $x_{r} \in F i c(h)$. Assume that $r \neq i$. By the same arguments as in the previous, we can show that $E s s(h) \subseteq E s s\left(f_{r \leftarrow s}\right)$. Because of 
$g a p(f)=p$, this implies $\operatorname{Ess}(h)=E s s\left(f_{r \leftarrow s}\right)$. Let us assume that $s \neq i$. Since $x_{r} \notin E s s(h)$ and $x_{i} \notin E s s(h)=E s s\left(f_{r \leftarrow s}\right)$, we have

$$
h=h_{r \leftarrow s} \quad \text { and } \quad f_{r \leftarrow s}=\left[f_{r \leftarrow s}\right]_{i \leftarrow l}
$$

for $l \in\{j, s\}$. Since $s \neq i$ and $r \neq i$, it is easy to see that $h_{r \leftarrow s}=\left[f_{r \leftarrow s}\right]_{i \leftarrow j}$ when $r \neq j$ and $h_{r \leftarrow s}=\left[f_{r \leftarrow s}\right]_{i \leftarrow s}$ when $r=j$. Altogether, this gives $f_{r \leftarrow s}=h$.

Next, let us assume that $s=i$. Since $x_{j} \notin E s s(h)=E s s\left(f_{r \leftarrow s}\right)$, we have $f_{r \leftarrow s}=\left[f_{r \leftarrow s}\right]_{j \leftarrow s}$. Moreover, $x_{j}, x_{r} \notin E s s(h)$ implies $h=\left[h_{r \leftarrow s}\right]_{j \leftarrow s}$. Since $s=i$, we have

$$
\left[f_{r \leftarrow s}\right]_{j \leftarrow s}=\left[h_{r \leftarrow s}\right]_{j \leftarrow s} \text { and thus } f_{r \leftarrow s}=h .
$$

Finally, assume that $r=i$ and $s \neq i$. Because of $x_{j} \notin E s s(h)$, we have in particular $h=f_{j \leftarrow i}$ where $x_{i} \notin E s s(h)=E s s\left(f_{j \leftarrow i}\right)$ (as we have shown in the previous). We might choose $\widetilde{h}:=f_{j \leftarrow i}$ (instead of $h:=f_{i \leftarrow j}$ ) and obtain $f_{j \leftarrow i}=$ $f_{i \leftarrow s}$ by the previous considerations. Altogether, we have $h=f_{i \leftarrow s}$.

Lemma 3.4. Let $f \in G_{p, k}^{n}$. Then the following conditions hold:

(i) If $2<p<n$ then there exist $u, v \in\{1, \ldots, n\}$ such that $f_{u \leftarrow v}$ depends essentially on $n-p$ variables and $x_{v} \in E s s\left(f_{u \leftarrow v}\right)$;

(ii) If $2<p \leq n$ then there exist $u, v \in\{1, \ldots, n\}$ such that $f_{u \leftarrow v}$ depends essentially on $n-p$ variables and $x_{v} \notin E s s\left(f_{u \leftarrow v}\right)$.

Proof. By Theorem 3.3, there are $u, v \in\{1, \ldots, n\}$ such that $\operatorname{ess}\left(f_{u \leftarrow v}\right)=n-p$ and $f_{u \leftarrow v}=f_{i \leftarrow j}$ for $1 \leq i, j \leq n$ with $i \neq j$ and $x_{i} \in F i c\left(f_{u \leftarrow v}\right)$.

(i) Since $p<n$, there is an $l \in\{1, \ldots, n\}$ with $x_{l} \in \operatorname{Ess}\left(f_{u \leftarrow v}\right)$. Then $f_{u \leftarrow v}=$ $f_{u \leftarrow l}$ since $x_{u} \in \operatorname{Fic}\left(f_{u \leftarrow v}\right)$. This shows $x_{l} \in \operatorname{Ess}\left(f_{u \leftarrow l}\right)$ where $\operatorname{ess}\left(f_{u \leftarrow l}\right)=n-p$.

(ii) It was already proved in Theorem 3.3

Theorem 3.4. Let $f$ be a $k$-valued function which depends essentially on the all of its $n$ variables and $2<p<n \leq k$. Then $f \in G_{p, k}^{n}$ if and only if there exist $n-p$ variables $y_{l_{1}}, \ldots, y_{l_{n-p}} \in X_{n}$ such that

$$
f=h \oplus g
$$

where $\operatorname{Ess}(h)=\left\{y_{l_{1}}, \ldots, y_{l_{n-p}}\right\}$ and $g \in G_{n, k}^{n}$. Moreover, $g_{i \leftarrow j}=0$ for all $1 \leq$ $i, j \leq n$ with $i \neq j$.

Proof. " $\Leftarrow "$ Let $f$ be represented in the form (5), where $h$ depends essentially on all of its $n-p$ variables and $g \in G_{n, k}^{n}$. With no loss of generality we might assume that $h$ is an $(n-p)$-ary $k$-valued function.

Again, without loss of generality, let us assume that $y_{l_{1}}, \ldots, y_{l_{n-p}}=x_{1}, \ldots, x_{n-p}$.

Since $2<p<n \leq k$ there is at least one variable $x_{j} \in X_{n}$ on which $h$ does not depend essentially i.e. $n-p<j \leq n$. Then from Lemma 2.2 we have $E s s\left(h_{j \leftarrow i}\right)=$ $\operatorname{Ess}(h)$ for all $i, 1 \leq i \leq n-p$. Since $g \in G_{n, k}^{n}$ it follows that $\operatorname{ess}\left(g_{u \leftarrow v}\right)=0$ for all $u$ and $v$ with $1 \leq u, v \leq n$ and $u \neq v$. Hence $\operatorname{ess}\left(f_{u \leftarrow v}\right)=e s s\left(h_{u \leftarrow v}\right)$ for all $u, v$ with $1 \leq u, v \leq n$ and $u \neq v$. On the other hand $\operatorname{ess}\left(h_{u \leftarrow v}\right)=n-p$ when $u>n-p$. Consequently,

$$
\max _{t \in \operatorname{Min}(f)} \operatorname{ess}(t)=n-p \text { and hence } g a p(f)=p .
$$

$" \Rightarrow "$ From Lemma 3.4 (ii) it follows that there are $u, v \in\{1, \ldots, n\}$ such that $f_{u \leftarrow v}$ depends essentially on $n-p$ variables and $x_{v} \notin E s s\left(f_{u \leftarrow v}\right)$. With no loss 
of generality let us assume that $(v, u)=(n-1, n)$ and let $h:=f_{n \leftarrow n-1}$, where $\operatorname{Ess}(h)=\left\{x_{1}, \ldots, x_{n-p}\right\}$.

Let $g \in P_{k}^{n}$ be the function defined by

$$
g:=f \ominus h
$$

i.e. $g$ is the unique function in $P_{k}^{n}$ such that $g \oplus h=f$.

We have to prove that $g \in G_{n, k}^{n}$ i.e. $\operatorname{Ess}(g)=X_{n}$ and $g_{i \leftarrow j}=0$ for all $i, j$ with $1 \leq j, i \leq n$ and $i \neq j$.

First, $\left\{x_{n-p+1}, \ldots, x_{n}\right\} \subseteq \operatorname{Ess}(g)$ because $\operatorname{Ess}(f)=X_{n}$ and $\left\{x_{n-p+1}, \ldots, x_{n}\right\} \cap$ $\operatorname{Ess}(h)=\emptyset$.

Second, to prove that $\left\{x_{1}, \ldots, x_{n-p}\right\} \subseteq E s s(g)$ we shall suppose that this is not the case and without loss of generality assume that $x_{1} \notin E s s(g)$. Then from Theorem 3.3 we obtain

$$
f_{n \leftarrow 1}=h\left(x_{1}, x_{2}, \ldots, x_{n-p}\right) \oplus g_{n \leftarrow 1}=h\left(x_{1}, x_{2}, \ldots, x_{n-p}\right) .
$$

Since $K$ is an additive group it follows that $g_{n \leftarrow 1}=0$. Consequently $g_{1 \leftarrow n}=0$ and from Lemma 2.2 we obtain $g=g_{1 \leftarrow n}=0$ which is a contradiction. Hence $\left\{x_{1}, \ldots, x_{n-p}\right\} \subseteq \operatorname{Ess}(g)$ and $\operatorname{Ess}(g)=X_{n}$.

To prove that $e s s\left(g_{i \leftarrow j}\right)=0$ for all $i, j$ with $1 \leq i, j \leq n$ and $i \neq j$, we shall consider several cases.

Case 1. Let $n-p<j, i<n$ and $i \neq j$. According to (66) we have $g_{n \leftarrow n-1}=$ $f_{n \leftarrow n-1} \ominus h=0$. From Theorem 3.3. it follows that $f_{i \leftarrow j}=f_{n \leftarrow n-1}$ and from Lemma 2.2 we have $h_{i \leftarrow j}=h$ which implies $g_{i \leftarrow j}=0$ i.e. ess $\left(g_{i \leftarrow j}\right)=0$ for all $i, j$ with $n-p<j, i<n$ and $i \neq j$.

Case 2. Let $j \leq n-p<i$. We may assume that $j=1$ and $i=n$. Since $x_{n} \notin E s s(h)$, we have $h=f_{n \leftarrow n-1}=f_{n \leftarrow 1}$ by Theorem 3.3. Moreover, from Lemma 2.2 we have $h=h_{n \leftarrow 1}$. Thus $g_{n \leftarrow 1}=f_{n \leftarrow 1} \ominus h_{n \leftarrow 1}=h \ominus h=0$. On the other hand we have

$$
h_{1 \leftarrow n}=\left[f_{n \leftarrow n-1}\right]_{1 \leftarrow n}=\left[f_{n \leftarrow 1}\right]_{1 \leftarrow n}=f_{1 \leftarrow n},
$$

i.e. $h_{1 \leftarrow n}=f_{1 \leftarrow n}$ and thus $g_{1 \leftarrow n}=f_{1 \leftarrow n} \ominus h_{1 \leftarrow n}=0$. Hence $g_{1 \leftarrow n}=g_{n \leftarrow 1}=0$.

Case 3. Let $i, j \leq n-p$ and $i \neq j$. By symmetry we may assume that $j=1$ and $i=2$. We have to prove that $g_{2 \leftarrow 1}=0$.

First let us assume that there is some $u>n-p$ such that $x_{u} \notin E s s\left(f_{2 \leftarrow 1}\right)$. According to Case 2 we have

$$
g_{u \leftarrow 1}=g_{u \leftarrow 2}=g_{1 \leftarrow u}=g_{2 \leftarrow u}=0 .
$$

From Lemma 3.3 we obtain $g_{2 \leftarrow u}=\left[g_{2 \leftarrow 1}\right]_{u \leftarrow 1}$ and Lemma 2.2 implies $g_{2 \leftarrow 1}=$ $\left[g_{2 \leftarrow 1}\right]_{u \leftarrow 1}$. Hence $g_{2 \leftarrow u}=g_{2 \leftarrow 1}=0$.

Second, assume that $\left\{x_{n-p+1}, \ldots, x_{n}\right\} \subseteq E s s\left(f_{2 \leftarrow 1}\right)$. Since $p \geq 3$ then $f \in G_{p, k}^{n}$ implies $\operatorname{ess}\left(f_{2 \leftarrow 1}\right)<n-2$.

Hence in the whole Case 3 the function $f_{2 \leftarrow 1}$ can essentially depend on at most $n-3$ variables. Then there is a variable $x_{v}, 3 \leq v \leq n-p$, which is not essential in $f_{2 \leftarrow 1}$. Without loss of generality let us assume that $v=3$. From Theorem 3.3 we have $f_{n-1 \leftarrow 3}=h$ and hence $f_{3 \leftarrow n-1}=h_{3 \leftarrow n-1}$. This implies $\left[f_{3 \leftarrow n-1}\right]_{2 \leftarrow 1}=$ $\left[h_{3 \leftarrow n-1}\right]_{2 \leftarrow 1}$. Hence $\left[f_{3 \leftarrow n-1}\right]_{2 \leftarrow 1}=\left[f_{2 \leftarrow 1}\right]_{3 \leftarrow n-1}=f_{2 \leftarrow 1}$ and $\left[h_{3 \leftarrow n-1}\right]_{2 \leftarrow 1}=$ $\left[\left[f_{2 \leftarrow 1}\right]_{3 \leftarrow n-1}\right]_{n \leftarrow n-1}$. From $f_{2 \leftarrow 1}=\left[f_{2 \leftarrow 1}\right]_{3 \leftarrow n-1}$ it follows

$$
\left[h_{3 \leftarrow n-1}\right]_{2 \leftarrow 1}=\left[f_{2 \leftarrow 1}\right]_{n \leftarrow n-1}=\left[f_{n \leftarrow n-1}\right]_{2 \leftarrow 1}=h_{2 \leftarrow 1} .
$$

Altogether, we have $f_{2 \leftarrow 1}=h_{2 \leftarrow 1}$, i.e. $g_{2 \leftarrow 1}=0$ and $\operatorname{ess}\left(g_{2 \leftarrow 1}\right)=0$. 
Consequently, $g \in G_{n, k}^{n}$ and $g_{i \leftarrow j}=0$ for all $1 \leq i, j \leq n$ with $i \neq j$.

Corollary 3.2. Let $f \in G_{p, k}^{n}$. Then there is a partition of the set $E s s(f)=$ $\left\{x_{1}, \ldots, x_{n}\right\}$ into the sets $V:=E s s(h)$ and $W:=E s s(f) \backslash V$, where $h$ is the function defined in the proof of Theorem 3.4, such that

$$
\left(x_{i}, x_{j}\right) \in W^{2} \Rightarrow\left(e s s\left(f_{i \leftarrow j}\right)=n-p \& x_{j} \notin E s s\left(f_{i \leftarrow j}\right)\right)
$$

and

$$
\left(x_{i}, x_{j}\right) \in W \times V \Rightarrow\left(e s s\left(f_{i \leftarrow j}\right)=n-p \& x_{j} \in \operatorname{Ess}\left(f_{i \leftarrow j}\right)\right) .
$$

Theorem 3.5. If $2<p<n \leq k$, then

$$
\left|G_{p, k}^{n}\right|=\left(k^{\left(\begin{array}{l}
k \\
n
\end{array}\right) \cdot n !}-1\right) \cdot \sum_{j=p}^{n}(-1)^{j-p}\left(\begin{array}{l}
j \\
p
\end{array}\right)\left(\begin{array}{l}
n \\
j
\end{array}\right) \cdot k^{k^{n-j}} .
$$

Proof. Let $f=h \oplus g \in G_{p, k}^{n}$ and $g_{i \leftarrow j}=0$ for all $1 \leq i, j \leq n$ with $i \neq j$. Let $a \in K$ be a non-zero natural number from $K$ i.e. $0<a \leq k-1$. Then clearly $t=g \oplus a \in G_{n, k}^{n}$ and $t_{i \leftarrow j}=a$ for all $1 \leq i, j \leq n$ with $i \neq j$. According to Theorem 3.2 the number of functions $g \in G_{n, k}^{n}$ with $g_{i \leftarrow j}=0$ for all $1 \leq i, j \leq n$ when $i \neq j$ is equal to $\left|G_{n, k}^{n}\right| / k=\left(k^{\left(\begin{array}{l}k \\ n\end{array}\right) \cdot n !}-1\right)$.

It is well known that the number of all functions in $P_{k}^{n}$ which depend essentially on exactly $n-p$ variables is equal to $\left|P_{n-p, k}^{n}\right|=\sum_{j=p}^{n}(-1)^{j-p}\left(\begin{array}{l}j \\ p\end{array}\right)\left(\begin{array}{l}n \\ j\end{array}\right) \cdot k^{k^{n-j}}$.

Consequently,

$$
\left|G_{p, k}^{n}\right|:=\left(\left|G_{n, k}^{n}\right| / k\right) \cdot\left(\left|P_{n-p, k}^{n}\right|\right)=\left(k^{\left(\begin{array}{c}
k \\
n
\end{array}\right) \cdot n !}-1\right) \cdot \sum_{j=p}^{n}(-1)^{j-p}\left(\begin{array}{c}
j \\
p
\end{array}\right)\left(\begin{array}{l}
n \\
j
\end{array}\right) \cdot k^{k^{n-j}} .
$$

\section{The Class $G_{2, k}^{n}$ WITH $4 \leq n \leq k$}

There are two subclasses of the class $G_{2, k}^{n}$ with $4 \leq n \leq k$. First one, consists of functions satisfying conditions similar as the conditions in Theorem 3.3 . Lemma 3.4 and Theorem 3.4 for $p=2$. The second subclass consists of functions whose behavior is similar to the functions from $G_{2, k}^{n}$ with $n>k$ (Theorem 2.1 in [15]).

Lemma 4.1. Let $f$ be a $k$-valued function which depends essentially on all of its $n, n>3$ variables and $g a p(f)=2$. Then there exist two distinct essential variables $x_{u}, x_{v}$ such that ess $\left(f_{u \leftarrow v}\right)=n-2$, and $x_{v} \notin E s s\left(f_{u \leftarrow v}\right)$. Moreover, $\operatorname{ess}\left(f_{u \leftarrow m}\right)=e s s\left(f_{v \leftarrow m}\right)=n-2$ for all $m, 1 \leq m \leq n$ with $m \notin\{u, v\}$.

Proof. Since $\operatorname{gap}(f)=2$, there are $1 \leq i, j \leq n$ with $i \neq j$ and $\operatorname{ess}\left(f_{i \leftarrow j}\right)=n-2$.

If $x_{j} \notin E s s\left(f_{i \leftarrow j}\right)$ we are done.

Let us assume that $x_{j} \in E s s\left(f_{i \leftarrow j}\right)$. Since $\operatorname{gap}(f)=2$, there is a $w \in\{1, \ldots, n\} \backslash$ $\{i, j\}$ such that $x_{w} \notin E s s\left(f_{i \leftarrow j}\right)$. From Lemma 3.2, we obtain

$$
\left[f_{w \leftarrow j}\right]_{i \leftarrow j}=f_{i \leftarrow j} \quad \text { and } \quad n-2=e s s\left(f_{i \leftarrow j}\right) \leq \operatorname{ess}\left(f_{w \leftarrow j}\right) .
$$

Hence $\operatorname{ess}\left(f_{i \leftarrow j}\right)=e s s\left(f_{w \leftarrow j}\right)=n-2$ since $\operatorname{gap}(f)=2$.

We shall prove that $x_{j} \notin E s s\left(f_{w \leftarrow j}\right)$ or $x_{i} \notin E s s\left(f_{w \leftarrow i}\right)$ which will complete the proof. 
For suppose this were not true. Then $x_{j} \in \operatorname{Ess}\left(f_{w \leftarrow j}\right)$ and $x_{i} \in \operatorname{Ess}\left(f_{w \leftarrow i}\right)$. Assume that $x_{i} \in \operatorname{Ess}\left(f_{w \leftarrow j}\right)$. Then there is a $r \in\{1, \ldots, n\} \backslash\{i, j, w\}$ such that $x_{r} \notin E s s\left(f_{w \leftarrow j}\right)$. Then $x_{r} \notin E s s\left(\left[f_{w \leftarrow j}\right]_{i \leftarrow j}\right)$, i.e. $x_{r} \notin E s s\left(f_{i \leftarrow j}\right)$, which is a contradiction. Hence $x_{i} \notin E s s\left(f_{w \leftarrow j}\right)$. By similar arguments, we obtain that $x_{j} \notin \operatorname{Ess}\left(f_{w \leftarrow i}\right)$.

Thus we have $\operatorname{Ess}\left(f_{i \leftarrow j}\right)=X_{n} \backslash\left\{x_{i}, x_{w}\right\}, \operatorname{Ess}\left(f_{w \leftarrow i}\right)=X_{n} \backslash\left\{x_{j}, x_{w}\right\}$ and $\operatorname{Ess}\left(f_{w \leftarrow j}\right)=X_{n} \backslash\left\{x_{i}, x_{w}\right\}$.

Since $n>3$ it follows that there is at least one essential variable $x_{s}$ in $f$ with $s \in\{1, \ldots, n\} \backslash\{i, j, w\}$.

Now, the equation $\operatorname{Ess}\left(f_{w \leftarrow s}\right)=X_{n} \backslash\left\{x_{w}\right\}$ is implicit in [15] (Lemma $\left.1.1(5)\right)$. This equation contradicts $\operatorname{gap}(f)=2$.

Let $m \notin\{u, v\}$ be a natural number with $1 \leq m \leq n$. Lemma 3.2 implies $f_{u \leftarrow v}=\left[f_{u \leftarrow m}\right]_{v \leftarrow m}$ for all $1 \leq m \leq n$ with $m \notin\{u, v\}$. Hence $n-2=\operatorname{ess}\left(f_{u \leftarrow v}\right)=$ $\operatorname{ess}\left(\left[f_{u \leftarrow m}\right]_{v \leftarrow m}\right) \leq \operatorname{ess}\left(f_{u \leftarrow m}\right)$. Now, gap $(f)=2$ shows that $\operatorname{ess}\left(f_{u \leftarrow m}\right)=n-2$ and by symmetry we obtain $\operatorname{ess}\left(f_{v \leftarrow m}\right)=n-2$

Let us denote by $G_{p, k}^{n,+}$ the set of all functions $f \in G_{p, k}^{n}$ for which there exist $i$ and $j$ with $1 \leq i, j \leq n$ such that $i \neq j, x_{j} \in \operatorname{Ess}\left(f_{i \leftarrow j}\right)$ and $\operatorname{ess}\left(f_{i \leftarrow j}\right)=n-p$.

$G_{p, k}^{n,-}$ denotes the set of all functions $f \in G_{p, k}^{n}$ for which $x_{v} \notin E s s\left(f_{u \leftarrow v}\right)$ for all $1 \leq u, v \leq n$ with $u \neq v$.

Proposition 4.1. If $3<n \leq k$ then $G_{2, k}^{n}=G_{2, k}^{n,+} \cup G_{2, k}^{n,-}$.

Proof. Clearly, $G_{2, k}^{n,+} \cup G_{2, k}^{n,-} \subseteq G_{2, k}^{n}$. Let $f \in G_{2, k}^{n}$. Then Lemma 4.1 implies that there exist two distinct essential variables $x_{u}, x_{v}$ such that $\operatorname{ess}\left(f_{u \leftarrow v}\right)=n-2$, and $x_{v} \notin \operatorname{Ess}\left(f_{u \leftarrow v}\right)$.

If $x_{j} \notin E s s\left(f_{i \leftarrow j}\right)$ for all $1 \leq i, j \leq n$ with $i \neq j$ then $f \in G_{2, k}^{n,-}$.

Next, assume that there are $1 \leq i, j \leq n$ with $x_{j} \in \operatorname{Ess}\left(f_{i \leftarrow j}\right)$. We have to prove that $f \in G_{2, k}^{n,+}$ i.e. there exist $r, s$ with $1 \leq r, s \leq n, x_{r} \in \operatorname{Ess}\left(f_{s \leftarrow r}\right)$ and ess $\left(f_{s \leftarrow r}\right)=n-2$.

If $\{i, j\} \cap\{u, v\} \neq \emptyset$ we are done because of Lemma 4.1

Let $\{i, j\} \cap\{u, v\}=\emptyset$. From Lemma 4.1 we have ess $\left(f_{u \leftarrow i}\right)=\operatorname{ess}\left(f_{u \leftarrow j}\right)=$ ess $\left(f_{v \leftarrow i}\right)=\operatorname{ess}\left(f_{v \leftarrow j}\right)=n-2$. If $x_{j} \in E s s\left(f_{u \leftarrow j}\right)$ we are done as above. If $x_{j} \notin \operatorname{Ess}\left(f_{i \leftarrow j}\right)$ then we have $x_{i} \in \operatorname{Ess}\left(f_{i \leftarrow j}\right)$ and $\operatorname{ess}\left(f_{u \leftarrow i}\right)=n-2$.

\section{Corollary 4.1.}

$$
G_{p, k}^{n}= \begin{cases}G_{p, k}^{n,+} & \text { if } \quad 2<p<n \leq k \\ G_{p, k}^{n,-} & \text { if } \quad(2 \leq n \leq k \& p=n) \quad \text { or }(n>k) \\ G_{p, k}^{n,+} \cup G_{p, k}^{n,-} & \text { if } \quad 3<n \leq k \& p=2 .\end{cases}
$$

Proof. This representation of the set $G_{p, k}^{n}$ follows by Theorem 3.4, Theorem 3.1] Proposition 4.1, Theorem 3.1 in [14] and Theorem 2.1 in [15].

\subsection{The subclass $G_{2, k}^{n,+}$.}

The first representation is related to Theorem 16 in [6]. 
Theorem 4.1. Let $4 \leq n \leq k$ and $f \in P_{k}^{n}$. Then the following statements are equivalent:

(i) $f \in G_{2, k}^{n,+}$;

(ii) There is a function $h \in P_{k}^{n}$ with ess $(h)=n-2$ and $f_{r \leftarrow s}=h$ for all $1 \leq r, s \leq n$ with $r \neq s$ and $x_{r} \in$ Fic $(h)$.

Proof. $(i i) \Rightarrow(i)$ is clear.

$(i) \Rightarrow(i i)$. Let $i$ and $j$ be two distinct natural numbers fro which $1 \leq i, j \leq n$, $x_{j} \in \operatorname{Ess}\left(f_{i \leftarrow j}\right)$ and $e s s\left(f_{i \leftarrow j}\right)=n-2$. Following the proof of Lemma 4.1 we might conclude that there exists an essential variable $x_{w}$ in $f$ such that $x_{w} \notin E s s\left(f_{i \leftarrow j}\right)$, $e s s\left(f_{w \leftarrow i}\right)=e s s\left(f_{w \leftarrow j}\right)=n-2$, and $x_{i} \notin E s s\left(f_{w \leftarrow i}\right)$ or $x_{j} \notin E s s\left(f_{w \leftarrow j}\right)$. Without loss of generality let us assume that $x_{i} \notin E s s\left(f_{w \leftarrow i}\right)$ i.e. $\operatorname{Ess}\left(f_{w \leftarrow i}\right)=X_{n} \backslash\left\{x_{w}, x_{i}\right\}$.

From Lemma 4.1 we have ess $\left(f_{w \leftarrow r}\right)=e s s\left(f_{i \leftarrow r}\right)=n-2$ for all $r, 1 \leq r \leq n$ with $r \notin\{w, i\}$.

First, we shall show that $X_{n} \backslash\left\{x_{w}, x_{i}, x_{r}\right\} \subset \operatorname{Ess}\left(f_{w \leftarrow r}\right)$ and $X_{n} \backslash\left\{x_{w}, x_{i}, x_{r}\right\} \subset$ $E s s\left(f_{i \leftarrow r}\right)$ for all $r, 1 \leq r \leq n$ with $r \notin\{w, i\}$. Note that $X_{n} \backslash\left\{x_{w}, x_{i}, x_{r}\right\} \neq \emptyset$ because $n>3$. Since $x_{i} \notin E s s\left(f_{w \leftarrow i}\right)$ and from Lemma 3.3 it follows

$$
f_{w \leftarrow i}=\left[f_{w \leftarrow r}\right]_{i \leftarrow r}=\left[f_{w \leftarrow i}\right]_{i \leftarrow r} .
$$

Let $x_{s}$ be an essential variable in $f$ with $s \notin\{w, i, r\}$. Suppose that $x_{s} \notin E s s\left(f_{w \leftarrow r}\right)$. Hence $x_{s} \notin E s s\left(\left[f_{w \leftarrow i}\right]_{i \leftarrow r}\right)$ i.e. $x_{s} \notin E s s\left(f_{w \leftarrow i}\right)$, which contradicts $E s s\left(f_{w \leftarrow i}\right)=$ $X_{n} \backslash\left\{x_{w}, x_{i}\right\}$.

Second, we shall prove that $x_{r} \in \operatorname{Ess}\left(f_{w \leftarrow r}\right)$ and $x_{r} \in \operatorname{Ess}\left(f_{i \leftarrow r}\right)$ for all $r$, $1 \leq r \leq n$ with $r \notin\{w, i\}$. Since $x_{j} \in E s s\left(f_{i \leftarrow j}\right)$ it follows that $j \notin\{w, i\}$.

Consider the case $r=j$. We have known that $x_{j} \in \operatorname{Ess}\left(f_{i \leftarrow j}\right)$ and from $x_{w} \notin$ $\operatorname{Ess}\left(f_{i \leftarrow j}\right)$ we obtain

$$
f_{i \leftarrow j}=\left[f_{i \leftarrow j}\right]_{w \leftarrow j}=\left[f_{i \leftarrow w}\right]_{w \leftarrow j}=f_{i \leftarrow w} .
$$

Hence $x_{j} \in \operatorname{Ess}\left(f_{w \leftarrow j}\right)$.

Assume that $r \notin\{w, i, j\}$. Suppose that $x_{r} \notin E s s\left(f_{i \leftarrow r}\right)$ and from Lemma 3.3 we have $f_{i \leftarrow r}=\left[f_{i \leftarrow j}\right]_{r \leftarrow j}$. Since $x_{w} \notin E s s\left(f_{i \leftarrow j}\right)$ we have $x_{w} \notin E s s\left(\left[f_{i \leftarrow j}\right]_{r \leftarrow j}\right)$ i.e. $x_{w} \notin E s s\left(f_{i \leftarrow r}\right)$. Then $\operatorname{ess}\left(f_{i \leftarrow r}\right)=n-3$ because $x_{r}, x_{i}, x_{w} \notin E s s\left(f_{i \leftarrow r}\right)$ which is a contradiction. Hence $x_{r} \in E s s\left(f_{i \leftarrow r}\right)$. In a similar way it might be shown that $x_{r} \in \operatorname{Ess}\left(f_{w \leftarrow r}\right)$.

Hence $\operatorname{Ess}\left(f_{i \leftarrow r}\right)=\operatorname{Ess}\left(f_{w \leftarrow r}\right)=E s s\left(f_{w \leftarrow i}\right)$ for all $r, 1 \leq r \leq n$ with $r \notin\{w, i\}$.

Finally, let us set $h:=f_{w \leftarrow i}$. Clearly $E s s(h)=X_{n} \backslash\left\{x_{w}, x_{i}\right\}$ and $\operatorname{ess}(h)=n-2$. Let $r$ and $s$ be two natural numbers such that $1 \leq r, s \leq n, r \neq s$ and $r \in\{w, i\}$. With no loss of generality let us assume that $r=w$. Since $x_{i} \notin E s s\left(f_{r \leftarrow i}\right)$ then Lemma 3.3 implies

$$
h=f_{r \leftarrow i}=\left[f_{r \leftarrow s}\right]_{i \leftarrow s}=\left[f_{i \leftarrow s}\right]_{r \leftarrow s}=f_{r \leftarrow s},
$$

because $x_{i} \notin E s s\left(f_{r \leftarrow s}\right)$.

Corollary 4.2. If $f \in G_{2, k}^{n,+}, n>3$ then there exist $x_{u}, x_{v} \in \operatorname{Ess}(f)$ such that $f_{r \leftarrow s}=f_{u \leftarrow v}=h$ for all $r \in\{u, v\}$ and $s \in\{1, \ldots, n\}, s \neq r$, as well as $\operatorname{ess}\left(f_{u \leftarrow v}\right)=n-2$.

Theorem 4.2. Let $f$ be a k-valued function which depends essentially on the all of its $n$ variables , $n>3$. Then the following sentences are equivalent:

(i) $f \in G_{2, k}^{n,+}$; 
(ii) There exist $n-2$ variables $y_{l_{1}}, \ldots, y_{l_{n-2}} \in X_{n}$ such that

$$
f=h \oplus g,
$$

where Ess $(h)=\left\{y_{l_{1}}, \ldots, y_{l_{n-2}}\right\}$ and $g \in G_{n, k}^{n}$. Moreover $g_{i \leftarrow j}=0$ for all $1 \leq i, j \leq$ $n$ with $i \neq j$.

Proof. We might prove the theorem in a similar way as Theorem 3.4 by using Theorem 4.1 instead of Theorem 3.3 .

Proposition 4.2. $\left|G_{2, k}^{n,+}\right|=\left(k^{\left(\begin{array}{c}k \\ n\end{array}\right) \cdot n !}-1\right) \cdot \sum_{j=2}^{n}(-1)^{j}\left(\begin{array}{c}j \\ 2\end{array}\right)\left(\begin{array}{c}n \\ j\end{array}\right) \cdot k^{k^{n-j}}$.

The proof can be done in a similar way as the proof of Theorem 3.5 .

\subsection{The subclass $G_{2, k}^{n,-}$.}

Thus Lemma 4.1 implies $G_{2, k}^{n,+} \cup G_{2, k}^{n,-}=G_{2, k}^{n}$. We are going to describe the class $G_{2, k}^{n,-}$ when $3<n \leq k$.

The next theorem is proved for $n>k$ by $\mathrm{R}$. Willard in [15].

Theorem 4.3. Let $f$ be a $k$-valued function which depends essentially on all of its $n, n>3$ variables. If $f \in G_{2, k}^{n,-}$ then each identification minor of $f$ is a symmetric function with respect to its essential variables.

Proof. Since $G_{2, k}^{n,-} \subset G_{2, k}^{n}$ then Lemma 4.1 implies that there exist two distinct essential variables $x_{u}, x_{v}$ such that $\operatorname{ess}\left(f_{u \leftarrow v}\right)=n-2$, and $x_{v} \notin \operatorname{Ess}\left(f_{u \leftarrow v}\right)$. With no loss of generality let us assume that $(v, u)=(1,2)$ and it is enough to prove that $f_{2 \leftarrow 1}$ is a symmetric function with respect to the variables from the set $\operatorname{Ess}\left(f_{2 \leftarrow 1}\right)$. Since $E s s\left(f_{2 \leftarrow 1}\right)=\left\{x_{3}, \ldots, x_{n}\right\}$ there is an $n-2$-ary function $h: K^{n-2} \rightarrow K$ such that $f_{2 \leftarrow 1}=h\left(x_{3}, \ldots, x_{n}\right)$. By symmetry, we have to prove that

$$
h\left(x_{3}, x_{4}, x_{5}, \ldots, x_{n}\right)=h\left(x_{4}, x_{3}, x_{5}, \ldots, x_{n}\right) .
$$

In fact, we obtain

$$
\begin{array}{ll}
h\left(x_{3}, x_{4}, x_{5} \ldots, x_{n}\right)= & \\
=f\left(x_{1}, x_{1}, x_{3}, x_{4}, x_{5}, \ldots, x_{n}\right) & \\
=f\left(x_{3}, x_{3}, x_{3}, x_{4}, x_{5}, \ldots, x_{n}\right) & \left(\text { since } x_{1} \notin \operatorname{Ess}\left(f_{2 \leftarrow 1}\right)\right. \\
=f\left(x_{4}, x_{3}, x_{4}, x_{4}, x_{5}, \ldots, x_{n}\right) & \left(\text { since } x_{3} \notin \operatorname{Ess}\left(f_{1 \leftarrow 3}\right)\right. \\
=f\left(x_{4}, x_{3}, x_{3}, x_{3}, x_{5}, \ldots, x_{n}\right) & \left(\text { since } x_{4} \notin \operatorname{Ess}\left(f_{3 \leftarrow 4}\right)\right. \\
=f\left(x_{4}, x_{4}, x_{4}, x_{3}, x_{5}, \ldots, x_{n}\right) & \left(\text { since } x_{3} \notin \operatorname{Ess}\left(f_{2 \leftarrow 3}\right)\right. \\
=f\left(x_{1}, x_{1}, x_{4}, x_{3}, x_{5}, \ldots, x_{n}\right) & \left(\text { since } x_{1} \notin \operatorname{Ess}\left(f_{2 \leftarrow 1}\right)\right. \\
=h\left(x_{4}, x_{3}, x_{5}, \ldots, x_{n}\right) . &
\end{array}
$$

We can now give a representation of the functions in $G_{2, k}^{n,-}$ (see also Theorem 16 in 6])

Theorem 4.4. Let $f$ be an $n$-ary $k$-valued function with $3<n \leq k$. Then the following sentences are equivalent:

(i) $f \in G_{2, k}^{n,-}$;

(ii) $f=t \oplus g$ where $g \in G_{n, k}^{n}$ and $t$ is an n-ary totally symmetric function with $\operatorname{Ess}\left(t_{i \leftarrow j}\right)=X_{n} \backslash\left\{x_{i}, x_{j}\right\}$ for all $i, j \in\{1, \ldots, n\}, i \neq j$. Moreover $g_{i \leftarrow j}=0$ for all $1 \leq i, j \leq n$ with $i \neq j$. 
Proof. " $\Leftarrow "$ Clearly, ess $\left(t_{i \leftarrow j}\right)=n-2$ and $\operatorname{ess}\left(g_{i \leftarrow j}\right)=0$ for all $i, j \in\{1, \ldots, n\}$, $i \neq j$. Hence $\operatorname{ess}\left(f_{i \leftarrow j}\right)=n-2$ and $g a p(f)=2$. We have to prove that $E s s(f)=$ $X_{n}$. By symmetry it is enough to show that $x_{1} \in E s s(t \oplus g)$. Since $n>3$ we have

$$
f\left(x_{1}, x_{2}, x_{2}, x_{4}, \ldots, x_{n}\right)=f_{3 \leftarrow 2}=t_{3 \leftarrow 2} \oplus g_{3 \leftarrow 2}
$$

and

$$
\begin{aligned}
& \operatorname{Ess}\left(f_{3 \leftarrow 2}\right)=E s s\left(t_{3 \leftarrow 2} \oplus g_{3 \leftarrow 2}\right)=\left\{x_{1}, x_{4}, \ldots, x_{n}\right\}=\operatorname{Ess}\left(t_{3 \leftarrow 2}\right) \subseteq \operatorname{Ess}(f) . \\
& \Rightarrow " \text { Let } f \in G_{2, k}^{n} . \text { Let us set } f=t \oplus g, \text { where } \\
t= & \bigoplus_{\alpha_{1} \alpha_{2} \ldots \alpha_{n} \in E q_{k}^{n}} a_{m} \cdot x_{1}^{\alpha_{1}} x_{2}^{\alpha_{2}} \ldots x_{n}^{\alpha_{n}} \quad \text { and } \quad g=\bigoplus_{\beta_{1} \beta_{2} \ldots \beta_{n} \notin E q_{k}^{n}} a_{r} \cdot x_{1}^{\beta_{1}} x_{2}^{\beta_{2}} \ldots x_{n}^{\beta_{n}},
\end{aligned}
$$

with $m=\overline{\alpha_{1} \alpha_{2} \ldots \alpha_{n}}$ and $r=\overline{\beta_{1} \beta_{2} \ldots \beta_{n}}$.

Note that such representation of $f$ can be obtained after a suitable reordering of the conjunctions in its $S C$-form.

Clearly, $g_{i \leftarrow j}=0$ for all $i, j \in\{1, \ldots, n\}, i \neq j$.

From Theorem 4.3 it follows that $f_{i \leftarrow j}$ is totally symmetric and $\operatorname{Ess}\left(f_{i \leftarrow j}\right)=$ $X_{n} \backslash\left\{x_{i}, x_{j}\right\}$ for all $i, j \in\{1, \ldots, n\}, i \neq j$. Since $f_{i \leftarrow j}=t_{i \leftarrow j}$ from Theorem 4.3 we can conclude that $t_{i \leftarrow j}$ is totally symmetric. We have to show that $t$ is totally symmetric, also. Since $\operatorname{Ess}\left(t_{i \leftarrow j}\right)=X_{n} \backslash\left\{x_{i}, x_{j}\right\}$ there is an $n-2$-ary function $h$ such that $t_{i \leftarrow j}=h$, where $h$ is a totally symmetric function (according to Theorem 4.3) which depends essentially on all of its variables. By $t\left(\alpha_{1}, \ldots, \alpha_{n}\right)=0$ when $\alpha_{1} \ldots \alpha_{n} \notin E q_{k}^{n}$ it suffices to prove that

$$
\begin{array}{r}
t\left(\alpha_{1}, \ldots, \alpha_{j-1}, \beta, \alpha_{j+1}, \ldots, \alpha_{i-1}, \beta, \alpha_{i+1}, \ldots, \alpha_{n}\right)= \\
h\left(\alpha_{1}, \ldots, \alpha_{j-1}, \alpha_{j+1}, \ldots, \alpha_{i-1}, \alpha_{i+1}, \ldots, \alpha_{n}\right),
\end{array}
$$

for all $i, j \in\{1, \ldots, n\}, i \neq j$.

First we shall prove (77) for $j=1$ and $i=3$. Since $x_{1} \notin E s s\left(f_{3 \leftarrow 1}\right)$ we obtain

$$
t\left(\alpha_{1}, \alpha_{2}, \alpha_{1}, \ldots, \alpha_{n}\right)=t\left(\alpha_{2}, \alpha_{2}, \alpha_{2}, \ldots, \alpha_{n}\right)=h\left(\alpha_{2}, \ldots, \alpha_{n}\right)
$$

as desired.

In a similar way we might show (7) for $j=2$ and $i=3$. As in the proof of Theorem 4.3 we may reorder the variables and show (7) for all $i, j \in\{1, \ldots, n\}$, $i \neq j$.

Corollary 4.3. Let $f \in G_{2, k}^{n}, 3<n<k$ and $x_{v} \notin E s s\left(f_{u \leftarrow v}\right)$ for all $v, u \in$ $\{1, \ldots, n\}, u \neq v$. Then there exists an $n-2$-ary totally symmetric function $h$ such that

$$
\begin{aligned}
f_{i \leftarrow j} & =f\left(x_{1}, \ldots, x_{j-1}, x_{j}, x_{j+1}, \ldots, x_{i-1}, x_{j}, x_{i+1}, \ldots, x_{n}\right) \\
& =h\left(x_{1}, \ldots, x_{j-1}, x_{j+1}, \ldots, x_{i-1}, x_{i+1}, \ldots, x_{n}\right),
\end{aligned}
$$

for all $i, j \in\{1, \ldots, n\}, i \neq j$.

Remark 4.1. We shall use some notation and results from [1] and [15].

Let $\operatorname{Sub}(K)$ denote the set of all subsets of $K$. Define oddsupp: $K^{n} \rightarrow \operatorname{Sub}(K)$ as follows

$$
\text { oddsupp }\left(\alpha_{1}, \ldots, \alpha_{n}\right):=\left\{\alpha_{v}:\left|\left\{u: 1 \leq u \leq n \& \alpha_{u}=\alpha_{v}\right\}\right| \text { is odd }\right\} .
$$

A function $f$ is determined by oddsupp if there exists a function $f^{*}: \operatorname{Sub}(K) \rightarrow$ $K$ such that $f=f^{*} \circ$ oddsupp. It is easy to see that the functions which are 
determined by oddsupp are totally symmetric, too. Then from Theorem 16 [6] it follows that the function $t$ defined in Theorem 4.4 is determined by oddsupp.

\section{The Class $G_{2, k}^{n}$ WITH $2 \leq n \leq 3 \leq k$}

Let us note that the class $G_{2, k}^{2}$ is described in Section 3 by Theorem 3.1 Thus $f \in G_{2, k}^{2}$ if and only if

$$
f=\left[\bigoplus_{\alpha \neq \beta} a_{r} x_{1}^{\alpha} x_{2}^{\beta}\right] \oplus a_{0} \cdot\left(x_{1}^{0} x_{2}^{0} \oplus \ldots \oplus x_{1}^{k-1} x_{2}^{k-1}\right)
$$

where at least two among the coefficients $\left\{a_{0}\right\} \cup\left\{a_{r} \mid r=\overline{\alpha \beta}, \& \alpha \neq \beta\right\}$ are distinct.

Example 5.1. Let $f \in P_{3}^{3}$ be the following function

$$
f=x_{1}^{0} x_{2}^{0} x_{3}^{0} \oplus x_{1}^{0} x_{2}^{0} x_{3}^{1} \oplus x_{1}^{0} x_{2}^{0} x_{3}^{2} \oplus x_{1}^{0} x_{2}^{1} x_{3}^{0} \oplus x_{1}^{0} x_{2}^{2} x_{3}^{0} \oplus x_{1}^{1} x_{2}^{0} x_{3}^{0} \oplus x_{1}^{2} x_{2}^{0} x_{3}^{0} .
$$

It is easy to check that $f_{2 \leftarrow 1}=f_{3 \leftarrow 1}=x_{1}^{0}, f_{3 \leftarrow 2}=x_{2}^{0}$ and hence $f \in G_{2,3}^{3}$.

Let $g \in P_{3}^{3}$ be the following function

$$
g=x_{1}^{0} x_{2}^{0} x_{3}^{0} \oplus x_{1}^{0} x_{2}^{1} x_{3}^{1} \oplus x_{1}^{0} x_{2}^{2} x_{3}^{2} \oplus x_{1}^{1} x_{2}^{0} x_{3}^{1} \oplus x_{1}^{1} x_{2}^{1} x_{3}^{0} \oplus x_{1}^{2} x_{2}^{0} x_{3}^{2} \oplus x_{1}^{2} x_{2}^{2} x_{3}^{0} .
$$

It is not difficult to see that $g_{2 \leftarrow 1}=x_{3}^{0}, g_{3 \leftarrow 1}=x_{2}^{0}, g_{3 \leftarrow 2}=x_{1}^{0}$ and hence $g \in G_{2,3}^{3}$.

Let $h \in P_{3}^{3}$ be the following function

$$
h=x_{1}^{0} x_{2}^{0} \oplus x_{1}^{0} x_{2}^{1} x_{3}^{1} \oplus x_{1}^{0} x_{2}^{2} x_{3}^{2} \oplus x_{1}^{1} x_{2}^{0} x_{3}^{1} \oplus x_{1}^{2} x_{2}^{0} x_{3}^{2} .
$$

It is clear that $h_{2 \leftarrow 1}=h_{3 \leftarrow 2}=x_{1}^{0}, h_{3 \leftarrow 1}=x_{2}^{0}$ and hence $h \in G_{2,3}^{3}$.

Let $r \in P_{3}^{3}$ be the following function

$$
r=x_{2}^{0} \oplus 2 . x_{1}^{1} x_{2}^{0} x_{3}^{2} \oplus 2 . x_{1}^{2} x_{2}^{0} x_{3}^{1} .
$$

It is clear that $r_{3 \leftarrow 1}=r_{3 \leftarrow 2}=x_{2}^{0}, r_{2 \leftarrow 1}=x_{1}^{0}$ and hence $r \in G_{2,3}^{3}$.

Clearly, the functions $f, g$ and $h$ do not satisfy Theorem 4.1 for $n=3$, and the functions $f, h$, and $r$ do not satisfy Theorem 4.4 for $n=3$, but $g \in G_{2,3}^{3,-}$, and $r \in G_{2,3}^{3,+}$.

Example 5.1 shows that the case $(p, n)=(2,3)$ is a special case in studying the functions with non-trivial arity gap.

In this section we shall pay attention to description of the class $G_{2, k}^{3}$ with $k>2$.

Lemma 5.1. If $f \in G_{2, k}^{3}$ then ess $\left(f_{i \leftarrow j}\right)=1$ for all $i, j \in\{1,2,3\}, i \neq j$.

Proof. Since $f \in G_{2, k}^{3}$ there is an identification minor of $f$ which depends essentially on one variable and let us suppose $\operatorname{ess}\left(f_{2 \leftarrow 1}\right)=1$. There are two possibilities.

(i) $\operatorname{Ess}\left(f_{2 \leftarrow 1}\right)=\left\{x_{3}\right\}$. Then there are three constants $\alpha_{1}, \alpha_{3}, \beta_{3} \in K$ such that $f\left(\alpha_{1}, \alpha_{1}, \alpha_{3}\right) \neq f\left(\alpha_{1}, \alpha_{1}, \beta_{3}\right)$.

(ii) $\operatorname{Ess}\left(f_{2 \leftarrow 1}\right)=\left\{x_{1}\right\}$. Then there exist three constants $\mu_{1}, \mu_{2}, \nu_{3} \in K$, such that $f\left(\mu_{1}, \mu_{1}, \nu_{3}\right) \neq f\left(\mu_{2}, \mu_{2}, \nu_{3}\right)$.

Now let us suppose the lemma is false and $\operatorname{ess}\left(f_{3 \leftarrow 1}\right)=0$. This implies that for all four constants $\delta_{1}, \varepsilon_{1}, \sigma_{2}, \psi_{2} \in K$ we have $f\left(\delta_{1}, \sigma_{2}, \delta_{1}\right)=f\left(\varepsilon_{1}, \psi_{2}, \varepsilon_{1}\right)$ which contradicts the both inequalities, listed above in $(i)$ and (ii). The similar arguments work if we suppose $\operatorname{ess}\left(f_{3 \leftarrow 2}\right)=0$. 
We are going to describe the properties of the functions from $G_{2,3}^{3}$. For this we need the following auxiliary functions.

$$
\begin{aligned}
& s\left(x_{1}, x_{2}\right):=\bigoplus_{\beta=\alpha} x_{1}^{\beta} x_{2}^{\alpha}, u^{(\alpha)}\left(x_{1}, x_{2}\right):=\bigoplus_{\beta \neq \alpha} x_{1}^{\beta} x_{2}^{\beta}, \\
& v^{(\alpha)}\left(x_{1}, x_{2}\right):=\bigoplus_{\beta \neq \alpha} x_{1}^{\alpha} x_{2}^{\beta}
\end{aligned}
$$

For example $u^{(1)}\left(x_{3}, x_{2}\right)=x_{3}^{0} x_{2}^{0} \oplus x_{2}^{2} x_{2}^{2}$ and $v^{(1)}\left(x_{3}, x_{2}\right)=x_{3}^{1} x_{2}^{0} \oplus x_{3}^{1} x_{2}^{2}$.

The following representation of the elements in $G_{2,3}^{3}$ should be also compared with Theorem 17(iii) in [6].

Theorem 5.1. If $f \in G_{2,3}^{3}$, then $f$ has to be represented in one of the following special forms, up to permutation of variables:

$$
f=\bigoplus_{i=0}^{2} a_{0}^{(i)}\left[x_{3}^{i} . s\left(x_{1}, x_{2}\right) \oplus x_{2}^{i} \cdot u^{(i)}\left(x_{1}, x_{3}\right) \oplus x_{1}^{i} \cdot u^{(i)}\left(x_{2}, x_{3}\right)\right] \oplus p_{3}\left(x_{1}, x_{2}, x_{3}\right),
$$

$$
\begin{aligned}
& f=\bigoplus_{i=0}^{2} a_{0}^{(i)}\left[x_{1}^{i} \cdot x_{2}^{i} \oplus x_{1}^{i} \cdot u^{(i)}\left(x_{2}, x_{3}\right) \oplus x_{2}^{i} \cdot u^{(i)}\left(x_{1}, x_{3}\right)\right] \oplus p_{3}\left(x_{1}, x_{2}, x_{3}\right), \\
& f=\bigoplus_{i=0}^{2} a_{0}^{(i)}\left[x_{1}^{i} \cdot x_{2}^{i} \oplus x_{2}^{i} \cdot v^{(i)}\left(x_{3}, x_{1}\right) \oplus x_{2}^{i} \cdot u^{(i)}\left(x_{1}, x_{3}\right)\right] \oplus p_{3}\left(x_{1}, x_{2}, x_{3}\right), \\
& f=\bigoplus_{i=0}^{2} a_{0}^{(i)}\left[x_{1}^{i} \cdot x_{2}^{i} \oplus x_{1}^{i} \cdot v^{(i)}\left(x_{3}, x_{2}\right) \oplus x_{2}^{i} \cdot v^{(i)}\left(x_{3}, x_{1}\right)\right] \oplus p_{3}\left(x_{1}, x_{2}, x_{3}\right),
\end{aligned}
$$

such that at least two among the coefficients $a_{0}^{(0)}, a_{0}^{(1)}, a_{0}^{(2)}$ are different and $p_{3}$ are arbitrary functions defined by (4).

Proof. According to Lemma 5.1, for $f \in G_{2,3}^{3}$ we need ess $\left(f_{2 \leftarrow 1}\right)=\operatorname{ess}\left(f_{3 \leftarrow 1}\right)=$ $e s s\left(f_{3 \leftarrow 2)}=1\right.$. This is equivalent to the following conjunction of three disjunctions:

$\left(x_{1} \notin E s s\left(f_{2 \leftarrow 1}\right)\right.$ or $\left.x_{3} \notin E s s\left(f_{2 \leftarrow 1}\right)\right) \&\left(x_{1} \notin E s s\left(f_{3 \leftarrow 1}\right)\right.$ or $\left.x_{2} \notin E s s\left(f_{3 \leftarrow 1}\right)\right)$

$$
\&\left(x_{2} \notin E s s\left(f_{3 \leftarrow 2}\right) \text { or } x_{1} \notin E s s\left(f_{3 \leftarrow 2}\right)\right) \text {. }
$$

Thus we obtain the following linear systems of equations for the coefficients of the functions from $G_{2,3}^{3}$.

(a) $\quad\left(a_{0}^{(0)}, a_{0}^{(1)}, a_{0}^{(2)}\right)=\left(a_{4}^{(0)}, a_{4}^{(1)}, a_{4}^{(2)}\right)=\left(a_{8}^{(0)}, a_{8}^{(1)}, a_{8}^{(2)}\right)$

(b) $\left(a_{0}^{(0)}, a_{4}^{(0)}, a_{8}^{(0)}\right)=\left(a_{0}^{(1)}, a_{4}^{(1)}, a_{8}^{(1)}\right)=\left(a_{0}^{(2)}, a_{4}^{(2)}, a_{8}^{(2)}\right)$,

(a) $\quad\left(a_{0}^{(0)}, a_{1}^{(0)}, a_{2}^{(0)}\right)=\left(a_{3}^{(1)}, a_{4}^{(1)}, a_{5}^{(1)}\right)=\left(a_{6}^{(2)}, a_{7}^{(2)}, a_{8}^{(2)}\right)$

(b) $\left(a_{0}^{(0)}, a_{3}^{(1)}, a_{6}^{(2)}\right)=\left(a_{1}^{(0)}, a_{4}^{(1)}, a_{7}^{(2)}\right)=\left(a_{2}^{(0)}, a_{5}^{(1)}, a_{8}^{(2)}\right)$,

(a) $\quad\left(a_{0}^{(0)}, a_{3}^{(0)}, a_{6}^{(0)}\right)=\left(a_{1}^{(1)}, a_{4}^{(1)}, a_{7}^{(1)}\right)=\left(a_{2}^{(2)}, a_{5}^{(2)}, a_{8}^{(2)}\right)$

(b) $\quad\left(a_{0}^{(0)}, a_{1}^{(1)}, a_{2}^{(2)}\right)=\left(a_{3}^{(0)}, a_{4}^{(1)}, a_{5}^{(2)}\right)=\left(a_{6}^{(0)}, a_{7}^{(1)}, a_{8}^{(2)}\right)$. 
Now any solution is determined by a word (string) over the alphabetic $\{a, b\}$ with length 3 . For example let us consider the string $a b a$. Then we use equations $a$ from (13), $b$ from (14) and $a$ from (15).

Then we obtain

$$
\begin{aligned}
& a_{0}^{(0)}=a_{4}^{(0)}=a_{8}^{(0)}=a_{1}^{(0)}=a_{2}^{(0)}=a_{1}^{(1)}=a_{2}^{(2)} \\
& a_{0}^{(1)}=a_{4}^{(1)}=a_{8}^{(1)}=a_{3}^{(1)}=a_{5}^{(1)}=a_{3}^{(0)}=a_{5}^{(2)} \\
& a_{0}^{(2)}=a_{4}^{(2)}=a_{8}^{(2)}=a_{6}^{(2)}=a_{7}^{(2)}=a_{6}^{(0)}=a_{1}^{(1)} .
\end{aligned}
$$

Hence

$$
f=\bigoplus_{i=0}^{2} a_{0}^{(i)}\left[x_{1}^{i} \cdot x_{3}^{i} \oplus x_{1}^{i} \cdot u^{(i)}\left(x_{2}, x_{3}\right) \oplus x_{3}^{i} \cdot u^{(i)}\left(x_{1}, x_{2}\right)\right],
$$

i.e. $f$ is as the function presented by (10) where the variables $x_{2}$ and $x_{3}$ are permuted.

In this way we might generate $2^{3}=8$ linear systems for the coefficients.

The remaining seven cases of strings, might be checked in the same way. We will summarize the results. The functions in the form (9) are generated by the string $a a a$; (10) - by $a b a, b a a$ and $a a b$; (11) - by $a b b, b b a$ and $b a b$ and (12) - by $b b b$.

Proposition 5.1. $\left|G_{2,3}^{3}\right|=139968$.

Proof. Clearly, each of the functions in equations (9) - (12) can be written as follows

$$
f=\left[\bigoplus_{i=0}^{2} a_{0}^{(i)} \cdot g^{(i)}\left(x_{1}, x_{2}, x_{3}\right)\right] \oplus p_{3}\left(x_{1}, x_{2}, x_{3}\right),
$$

where $p_{3}$ are the functions defined in (4) when $k=3$. It is easy to show that the number of all such functions is equal to $3^{3 !}=729$.

The functions $g^{(i)}$ from the equations (9) and (12) are totally symmetric, but these from (10) and (11) are symmetric with respect to two of their arguments, only. Thus if the coefficients $a_{0}^{(i)}$ are fixed then there exist one function

$$
\bigoplus_{i=0}^{2} a_{0}^{(i)} \cdot g^{(i)}\left(x_{1}, x_{2}, x_{3}\right)
$$

determined by (9), one - by (12), three functions are determined by (10) and three - by (11). So, there are 8 such functions. The triples $\left(a_{0}^{(0)}, a_{0}^{(1)}, a_{0}^{(2)}\right)$ can be chosen in $3^{3}-3=24$ ways (the triples $(0,0,0),(1,1,1)$ and $(2,2,2)$ are forbidden). Hence there are $8.24=192$ functions $\bigoplus_{i=0}^{2} a_{0}^{(i)} \cdot g^{(i)}\left(x_{1}, x_{2}, x_{3}\right)$ which satisfied (9) - (12). Hence $\left|G_{2,3}^{3}\right|=192.729=139968$.

Proposition 5.2. $\left|G_{2, k}^{3}\right|=8.729 \cdot\left(\begin{array}{l}k \\ 3\end{array}\right) \cdot\left(k^{k}-k\right)=5832 \cdot\left(\begin{array}{l}k \\ 3\end{array}\right) \cdot\left(k^{k}-k\right)$.

Proof. Without any difficulties, excluding the more complex calculations, we might generalize results from $G_{2,3}^{3}$ to $G_{2, k}^{3}$ for arbitrary $k, k \geq 3$. In this case we obtain the same conjunction of three disjunctions to determine the functions from $G_{2, k}^{3}$. The difference is that in the equations (13)-15) participate $k$ tuples of $k$ coefficients and a function belongs to $G_{2, k}^{3}$ if and only if it can be represented as in (9)-(12) as the sums are extended up to $k-1$ instead of 2 in the case $G_{2,3}^{3}$. All other arguments work, here also. 


\section{ACKNOWLEDGEMENTS}

We are grateful to our colleagues K. Chimev, K. Denecke and D. Kovachev for useful discussions and their comments.

\section{REFERENCES}

[1] J. Berman and A. Kisielewicz, On the number of operations in a clone, Proc. Amer. Math Soc., 122 (1994), pp. 359-369.

[2] Yu. Breitbart, On the essential variables of functions in the algebra of logic, Dokl. Acad. Sci. USSR, 172, vol. 1, (1967), pp. 9-10 (in Russian).

[3] K. Chimev, Separable sets of arguments of functions, MTA SzTAKI Tanulmanyok, 180, (1986), $173 \mathrm{p}$.

[4] K. Chimev, On some properties of functions, Colloquia Mathematica Societatis Janos Bolyai, Szeged, (1981), pp. 97-110.

[5] M. Couceiro, E. Lehtonen, On the arity gap of finite functions: results and applications, Int. Conf. on Relations, Orders and Graphs: Interaction with Computer Science, Nouha Editions, Sfax, (2008), pp. 65-72, (http://www.math.tut.fi/algebra/papers/ROGICS08-CL.pdf).

[6] M. Couceiro, E. Lehtonen, Generalizations of Swierczkowski's lemma and the arity gap of finite functions, Discrete Mathematics, (2009), doi:10.1016/j.disc.2009.04.009.

[7] K. Denecke, J. Koppitz, Essential variables in hypersubstitutions, Algebra Universalis, 46 (2001), pp. 443-454

[8] D. Kovachev, On a class of discrete functions, Acta Cybernetica, vol. 17, No. 3, Szeged, (2006), pp. 513-519.

[9] O. Lupanov, On a class of schemes of functional elements, Problemi Kybernetiki, 9, (1963), pp. 333-335 (in Russian).

[10] A. Salomaa, On essential variables of functions, especially in the algebra of logic, Annales Academia Scientiarum Fennicae, Ser. A, 333, (1963), pp. 1-11.

[11] Sl. Shtrakov, K. Denecke, Essential variables and separable sets in universal algebra, Taylor \& Francis, Multiple-Valued Logic, vol. 8, No. 2, (2002), pp. 165-182.

[12] Sl. Shtrakov, Essential variables and positions in terms, Algebra Universalis, vol. 61, No. 3-4 (2009), pp. 381-397.

[13] Sl. Shtrakov, Tree automata and essential input variables, Contributions to General Algebra 13, Verlag Johannes Heyn, Klagenfurt, (2001), pp.309-320.

[14] Sl. Shtrakov, Essential arity gap of Boolean functions, Serdica Journal of Computing, vol.2, No. 3, (2008), pp. 249-266.

[15] R. Willard, Essential arities of term operations in finite algebras, Discrete Mathematics, 149 (1996), pp. 239-259.

Department of Computer Science, South-West University, 2700 Blagoevgrad, BulGARIA,

E-mail address: shtrakov@swu.bg

URL: http://home.swu.bg/shtrakov

Institute of Mathematics, University of Potsdam, 14415 Potsdam,

E-mail address: koppitz@rz.uni-potsdam.de 